Artículo científico

Volumen 31(1):105-115. Enero-abril, 2020

e-ISSN 2215-3608, doi:10.15517/am.v31i1.36866

http://www.revistas.ucr.ac.cr/index.php/agromeso

\title{
Aumento en la producción de okra con la adición de nitrógeno ${ }^{1}$
}

\section{Increase okra production with the addition of nitrogen}

\author{
Valdilene Coutinho-Miranda ${ }^{2}$, Irais Dolores Pascual-Reyes ${ }^{2}$, Aline Torquato-Tavares ${ }^{2}$, João Victor Gonçalves- \\ Carline $^{2}$, Kássio Abel Silva-Sousa ${ }^{2}$, Ildon Rodrigues-do-Nascimento ${ }^{2}$
}

1 Recepción: 22 de abril, 2019. Aceptación: 16 de setiembre, 2019. Este trabajo formó parte de la tesis de doctorado de la primera autora, realizada en la Universidade Federal do Tocantins, Programa de Posgrado en Producción Vegetal, Campus Gurupi, Brasil.

2 Universidade Federal do Tocantins, Programa de Pós Graduação em Produção Vegetal. Rua Badejós, Lote 7, Chácaras 69/72, Zona Rural. CEP 77402-970, Gurupi, Tocantins, Brasil. dilene.223@hotmail.com; irais121@hotmail.com (https://orcid.org/0000-0003-1192-9563); alinet4t@hotmail.com; jvictor92@hotmail.com; kassioabel.a@gmail.com; ildon@mail.uft.edu.br

\section{Resumen}

Introducción. El crecimiento, la producción y la calidad de okra (Abelmoschus esculentus (L.) Moench) están relacionados con la aplicación de fertilizantes, siendo el nitrógeno $(\mathrm{N})$ el que más destaca, debido a la relación directa con la fotosíntesis y crecimiento vegetativo de la planta. Objetivo. El objetivo fue evaluar las características agronómicas y de productividad de okra en función de la dosis de N. Materiales y métodos. El estudio se realizó en el área experimental Campus Gurupi, de la Universidad Federal de Tocantins (UFT), Brasil, en dos períodos de siembra (otoño/invierno y primavera/verano). El diseño experimental utilizado fue bloques al azar (DBC), con seis tratamientos $\left(0,50,100,150,200\right.$ y $\left.250 \mathrm{~kg} \mathrm{~N} \mathrm{ha}^{-1}\right)$ y cuatro repeticiones. Se utilizó urea como fuente de $\mathrm{N}$. Las características evaluadas fueron: productividad, masa media del fruto, altura e índice de clorofila de la planta. Resultados. La productividad y la altura de la planta resultaron superiores en el cultivo de otoño/invierno. La masa media e índice de clorofila no sufrieron influencia por el período de siembra. Para la productividad, se obtuvo una respuesta lineal en aumento de la dosis hasta el límite de la dosis de $\mathrm{N}$ utilizada $\left(250 \mathrm{~kg} \mathrm{ha}^{-1}\right)$, con valor medio superior a $14 \mathrm{t}$ de fruto. La masa media y la altura de la planta respondieron linealmente al aumento de la dosis de $\mathrm{N}$. El nitrógeno afectó el índice de clorofila, con valores máximos de 45,96 y 47,19, observados en los dos periodos de evaluación. Conclusión. La época de siembra y el contenido de $\mathrm{N}$ en el suelo interactuaron con la altura de la planta, siendo favorable en el periodo que no hubo precipitación. El N influyó en todas las características, lo que demuestra la importancia de la fertilización nitrogenada en el desarrollo de las plantas de okra.

Palabras claves: Abelmoschus esculentus, siembra, fertilización nitrogenada, respuesta lineal.

\begin{abstract}
Introduction. The growth, production, and quality of the okra (Abelmoschus esculentus (L.) Moench) are related to the application of fertilizers with nitrogen $(\mathrm{N})$ being the most prominent, due to the direct relationship with photosynthesis and vegetative growth of the plant. Objective. The objective was to evaluate the agronomic and productivity characteristics of the okra based on the dose of N. Materials and methods. The study was carried out in the experimental area Campus Gurupi, of the Universidad Federal de Tocantins (UFT), in two planting periods
\end{abstract}


(autumn/winter and spring/summer). The experimental design used was randomized blocks (DBC) with six treatments $\left(0,50,100,150,200\right.$, and $\left.250 \mathrm{~kg} \mathrm{~N} \mathrm{ha}^{-1}\right)$ and four repetitions. Urea was used as a source of $\mathrm{N}$. The characteristics evaluated were: productivity, average fruit mass, height and chlorophyll index of the plant. Results. The productivity and height of the plant were higher in the autumn/winter crop. The average mass and chlorophyll index were not influenced by the sowing period. For productivity a linear response was obtained, increasing the dose up to the limit of the dose of $\mathrm{N}$ used $\left(250 \mathrm{~kg} \mathrm{ha}^{-1}\right)$, with an average value higher than $14 \mathrm{t}$ of fruit. The mean mass and plant height responded linearly to the increase in the dose of N. Nitrogen affected the chlorophyll index, with maximum values of 45.96 and 47.19, observed in the two evaluation periods. Conclusion. The sowing season and the $\mathrm{N}$ content in the soil interacted with the height of the plant, being favorable in the period when there was no precipitation. $\mathrm{N}$ influenced all characteristics, demonstrating the importance of nitrogen fertilization development okra plants.

Keywords: Abelmoschus esculentus, sowing, nitrogenous fertilizing, linear response.

\section{Introducción}

La okra (Abelmoschus esculentus (L.) Moench) es una hortaliza de la familia de las malváceas, un alimento popular de alto valor nutricional, originaria de África e introducida a Brasil por los esclavos provenientes de este continente (Galati et al., 2013). Son plantas tolerantes al calor, presentan características deseables como ciclo rápido, producción económicamente viable y resistencia a plagas y enfermedades. En regiones de clima caliente puede producirse durante todo el año (Galati et al., 2013).

La okra es exigente en nitrógeno $(\mathrm{N})$, requerido en grandes cantidades por las plantas por estar presente en la composición de la producción de nuevas células y tejidos, tal como en el ATP, NADH, NADPH, en las moléculas de la clorofila, alcaloides, aminoácidos, proteínas, innumerables enzimas y vitaminas, hormonas y en varios compuestos del metabolismo de la planta (Nascimento et al., 2015).

En la okra, la fertilización nitrogenada desempeña un papel fundamental en la productividad de frutos comercializables. El $\mathrm{N}$ en el cultivo de la okra es un elemento esencial, pues promueve un aumento del índice de área foliar, lo que favorece el crecimiento vegetativo y eleva el número de yemas vegetativas y florales (Malavolta, 2008). El N también proporciona un aumento en la altura conforme al incremento en la dosis de fertilizante nitrogenado (Bredemeier y Mundstock, 2000).

Las recomendaciones de dosis de fertilización de $\mathrm{N}$ para okra son variables, desde $60 \mathrm{~kg} \mathrm{ha}^{-1}$ para la región del Amazonas hasta $180 \mathrm{~kg} \mathrm{ha}^{-1}$ para suelos con fertilidad media o baja (Cardoso, 2001).

Plantas crecidas con cantidades inadecuadas de $\mathrm{N}$ no expresan totalmente su potencial genético, debido a que ocurre una serie de alteraciones morfológicas, más energéticamente en los procesos fisiológicos en la planta, desde la absorción hasta la completa asimilación y transformación del $\mathrm{N}$ en moléculas orgánicas. Con dosis elevadas de N hay un efecto negativo en el crecimiento, las células desarrollan protoplasma más lento, no construyen material suficiente de apoyo para las paredes celulares, lo cual se torna en plantas frágiles y pueden ser vulnerables a daños mecánicos, de manera que se reduce su productividad (Taiz y Zeiger, 2013). Además, la deficiencia de $\mathrm{N}$ reduce el crecimiento, comprometiendo la partición de asimilados entre las diferentes partes de la planta, ocasionando generalmente aumento en la relación entre la masa seca de las raíces y de la parte aérea (Bredemeier y Mundstock, 2000).

A pesar de la gran importancia de la fertilización nitrogenada en el cultivo de la okra, poco se conoce al respecto de las mejores dosis a utilizar que permitan un buen desarrollo vegetativo y obtención de rendimientos satisfactorios, por lo cual, existe una necesidad de definir una dosis de $\mathrm{N}$ capaz de proporcionar rendimiento máximo, además de los otros beneficios que esa práctica proporciona al cultivo. 
El estado nutricional de las plantas, principalmente nitrogenado, está directamente asociado con la calidad y cantidad de clorofila, en razón de participar en la composición estructural de su molécula (Marcussi et al., 2004). Cuando hay deficiencia de N, un efecto directo que se observa es la reducción del índice de clorofila (Argenta, 2001; Chapman y Barreto, 1997).

Las dosis de $\mathrm{N}$ afectan la masa del fruto, pues la aplicación de $\mathrm{N}$ adecuado es primordial para el desarrollo, crecimiento, división, aumento, densidad celular y expansión fotosintética, siendo benéfico para el aumento de la masa del fruto, elevando la producción del cultivo (Filgueira, 2000).

Un aspecto básico de su manejo es conocer el momento adecuado para realizar la siembra de esta malvácea. diversos estudios realizados en varios países (Irán, Pakistán, México, Irak, Bangladesh, Suazilandia, India), muestran que el periodo de siembra (combinada con otros factores) influye de manera significativa en la emergencia, crecimiento y rendimiento del cultivo (Alvarado et al., 2007; Dilruba et al., 2009; Ossomon y Kunene, 2011).

Estudios realizados en Nigeria por Adejoye et al. (2009) concluyen que la okra puede sembrarse durante todo el año, siempre y cuando tenga agua disponible, especialmente durante la estación seca, para satisfacer sus requerimientos térmicos. El aumento de la temperatura provoca la reducción del ciclo en días para la apertura de la primera flor, aumenta la altura de la planta, reduce el número de ramas laterales e incrementa la productividad (Sonnenberg y Silva, 2002).

De acuerdo con Cerri y Vilella (1996) en Buenos Aires, las variedades Colhe Bem y Clemson Spineless, sembradas en el mes de abril, produjeron menos que cuando fueron plantadas en diciembre, debido a la helada que acortó el ciclo. En la India, en la región de Calcuta, las siembras de verano, realizadas durante la estación de primavera/verano (los meses de marzo a junio), resultaron con una mayor producción y con frutos de mejor calidad que los sembrados durante la estación de lluvia/rocío (los meses de julio a febrero) (Supatra-Sen y Sen, 1998).

El objetivo de este trabajo fue evaluar las características agronómicas y de productividad de la okra en función de la dosis de $\mathrm{N}$.

\section{Material y métodos}

El estudio se realizó durante el año 2015 en el área experimental del sector de olericultura de la Universidad Federal de Tocantins (UFT - Campus Gurupi), municipio de Gurupi, Estado de Tocantins, Brasil, localizada a

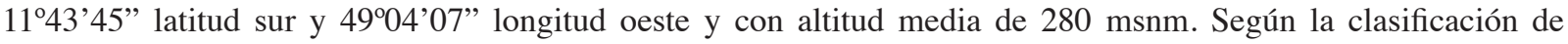
Köppen (1948), el clima de la región es Aw, definido como tropical de verano húmedo y período de sequía en invierno. Con una temperatura media anual de $29^{\circ} \mathrm{C}$ y una precipitación media anual de $1600 \mathrm{~mm}$ (INMET, 2015).

Las estimativas de temperatura, precipitación y humedad relativa del aire en el periodo de realización de los ensayos se presentan en la Figura 1.

Se establecieron dos experimentos, el primero se realizó de abril a julio (otoño/invierno) y el segundo de septiembre a diciembre (primavera/verano). El suelo se encuentra clasificado como litosol rojo-amarillo distrófico (Embrapa, 2013).

Se realizó análisis de suelo a una profundidad de 0-20 cm, presentando: $\mathrm{pH}$ en agua: 5,78; $\mathrm{H}+\mathrm{Al}: 1,46 \mathrm{cmol}$ $\mathrm{dm}^{-3}$; Ca+Mg: $4 \mathrm{cmol} \mathrm{dm}^{-3} ; \mathrm{K}: 0,23 \mathrm{cmol} \mathrm{dm}^{-3} ; \mathrm{P}: 26,01 \mathrm{mg} \mathrm{dm}^{-3}$; materia orgánica: 1,8; V: $74 \%$, arena total: 69,7 $\%$, limo: $5,72 \%$ y arcilla: $24,6 \%$.

El diseño experimental utilizado fue de bloques al azar con seis tratamientos $0,50,100,150,200$ y $250 \mathrm{~kg}$ $\mathrm{ha}^{-1}$ de $\mathrm{N}$ y cuatro repeticiones. Como fuente de $\mathrm{N}$ se utilizó urea (46-00-00). Las dosis de $\mathrm{N}$ estudiadas fueron distribuidas en dos aplicaciones, $50 \%$ se aplicó en el surco en el momento de la siembra y el $50 \%$ restante se agregó en la cobertura (30 días después de sembrar (dds)). 


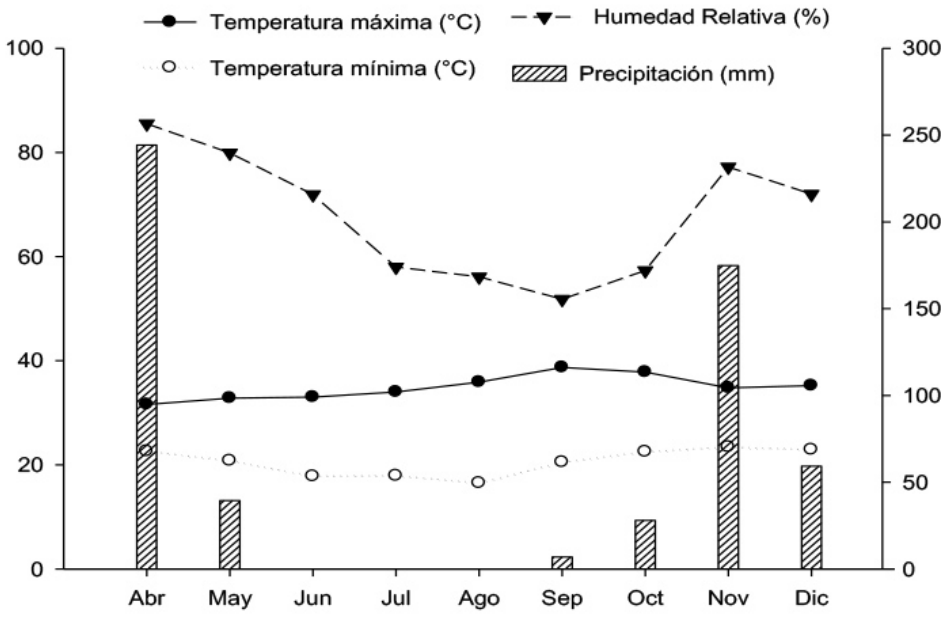

Figura 1. Valores promedios mensuales para temperatura del aire mínima y máxima $\left(\mathrm{en}{ }^{\circ} \mathrm{C}\right)$, humedad relativa del aire $(\mathrm{HR})$ y precipitación (en $\mathrm{mm}$ ), durante el periodo de conducción de los experimentos, para evaluar las características agronómicas y de productividad de la okra (Abelmoschus esculentus (L.) Moench) en función de la dosis de N. Campus Gurupi, Universidad Federal de Tocantins, Brasil, 2015. Fuente: INMET (2015).

Figure 1. Average monthly values for minimum and maximum air temperature (in ${ }^{\circ} \mathrm{C}$ ), relative humidity (RH) and rainfall (in mm) during the conduct of the experiments to evaluate the agronomic and productivity characteristics of the okra (Abelmoschus esculentus (L.) Moench) depending on the dose of N. Campus Gurupi, Universidad Federal de Tocantins, Brasil, 2015. Source: INMET (2015).

La fertilización se llevó acabo según el análisis del suelo. Se aplicó $80 \mathrm{~kg} \mathrm{ha}^{-1}$ de $\mathrm{K}_{2} \mathrm{O}$, distribuido en dos aplicaciones, $50 \%$ en la siembra y $50 \%$ en la cobertura (30 dds). Como fuente de fósforo se aplicó $60 \mathrm{~kg} \mathrm{ha}^{-1}$ de $\mathrm{P}_{2} \mathrm{O}_{5}$ en la siembra, en forma de superfosfato simple.

Las parcelas estuvieron compuestas por cuarenta plantas, distribuidas en cuatro surcos de 5,0 m de longitud, con espaciamiento de 0,50 m entre plantas y 0,80 m entre líneas, las veinte plantas centrales se consideraron como parcela útil. Se empleó la variedad Santa Cruz $47^{\circledR}$ en siembra directa, y se colocaron cuatro semillas por planta. Después de diez días de la emergencia se realizó un raleo a una planta por mata.

La preparación del suelo fue convencional. En el primer período (otoño/invierno) se realizó un riego por aspersión, en períodos de dos días hasta los 30 dds (totalizando quince irrigaciones) y con un período de tres días después de ese período hasta la cosecha (totalizando trece irrigaciones).

El control de malezas fue manual. Durante la conducción del cultivo, el control de plagas y enfermedades se realizó de acuerdo con la recomendación para el cultivo (Gonçalves, 2008).

La cosecha inició a los 45 días y, posteriormente se cosechó cada dos días, cuando los frutos se encontraban tiernos, sin fibras, coloración verde intenso y longitud por encima de $10 \mathrm{~cm}$. La duración de la cosecha fue de 45 días. Las características evaluadas fueron: 1) producción comercial del fruto: obtenida por el peso del fruto comercial cosechado de la parcela útil en una balanza digital y los datos convertidos en $\mathrm{Mg} \mathrm{ha}^{-1}$; 2) masa media del fruto comercial $(\mathrm{g})$ : obtenido por el peso del fruto comercial cosechado de la parcela útil en una balanza digital; 3) altura de la planta $(\mathrm{cm})$ : evaluada en en floración con una cinta métrica graduada en centímetros; 4) índice de clorofila total: determinado con el uso de un clorofilometro portátil modelo CFL 1030.

En cada período los valores medios de cada parcela se sometieron a análisis de varianza individual y conjunta (después de la comparación de la relación de los cuadrados medios del residuo), se aplicó la prueba de Tukey $(\mathrm{p} \leq 0,05)(1949)$ y un análisis de regresión. Las variables que tuvieron respuesta se ajustaron a modelos matemáticos para explicar la variación de los datos observados. Los gráficos se elaboraron en el programa Zigma Plot. Los análisis estadísticos se hicieron con el software SISVAR versión 5.3 (Ferreira, 2008). 


\section{Resultados}

Entre los períodos de evaluación, se encontraron diferencias significativas en productividad y altura de planta, no así, para el índice de clorofila y masa media del fruto (Cuadro 1).

Cuadro 1. Media general para productividad, altura de planta, clorofila, masa media, longitud, diámetro del fruto de la okra (Abelmoschus esculentus L.) en dos períodos siembra. Campus Gurupi, Universidad Federal de Tocantins, Brasil. 2015.

Table 1. The overall average for productivity, plant height, chlorophyll, average mass, length, diameter of the fruit of the okra (Abelmoschus esculentus L.) in two sowing periods. Campus Gurupi, Universidad Federal de Tocantins, Brasil. 2015.

\begin{tabular}{lcc}
\hline \multirow{2}{*}{ Características } & \multicolumn{2}{c}{ Período } \\
\cline { 2 - 3 } & Otoño/inverno & Primavera/verano \\
\hline Producción $\left(\mathrm{Mg} \mathrm{ha}^{-1}\right)$ & $13,86 \mathrm{a}$ & $9,95 \mathrm{~b}$ \\
Masa del fruto $(\mathrm{g})$ & $20,00 \mathrm{a}$ & $20,00 \mathrm{a}$ \\
Altura de planta (cm) & $92,46 \mathrm{a}$ & $71,06 \mathrm{~b}$ \\
Clorofila (ICF) & $44,04 \mathrm{a}$ & $44,48 \mathrm{a}$ \\
\hline
\end{tabular}

Medias seguidas de la misma letra minúscula en la línea indican que no difieren estadísticamente entre sí por la prueba de Tukey ( $\mathrm{p} \leq 0,05$ ). Means followed by the same lowercase letter on the line indicate that they do not differ statistically from each other by the Turkey test $(\mathrm{p} \leq 0.05)$.

A pesar que en la época de lluvia es más propenso el aparecimiento de insectos y patógenos, no se verificó ninguna incidencia, pues se realizaron aplicaciones de insecticida y fungicida visando al control de los mismos. Además de la fertilización, las condiciones climáticas locales son factores pre-cosecha que afectaron el rendimiento.

La productividad de la okra respondió a las dosis de $\mathrm{N}$ de forma lineal creciente (Figura 2), en que la dosis de $250 \mathrm{~kg} \mathrm{~h}^{-1}$ en los dos períodos fue la de máxima producción con 16,83 $\mathrm{Mg} \mathrm{ha}^{-1}$ (otoño/invierno) y 13,50 $\mathrm{Mg} \mathrm{ha}^{-1}$ (primavera/verano).

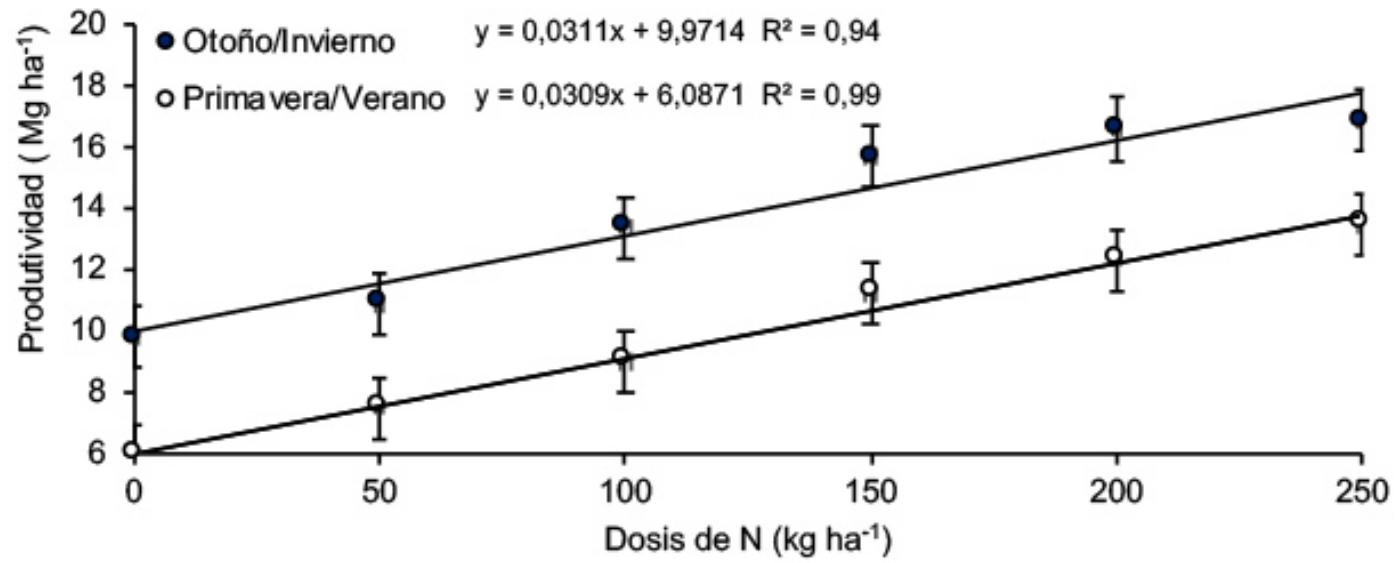

Figura 2. Productividad de fruto de la okra (Abelmoschus esculentus L.), en función de dosis de $\mathrm{N}$ en dos períodos de siembra. Campus Gurupi, Universidad Federal de Tocantins, Brasil. 2015.

Figure 2. Productivity of okra (Abelmoschus esculentus L.) fruit, depending on $\mathrm{N}$ dose in two sowing periods. Campus Gurupi, Universidad Federal de Tocantins. Brasil. 2015. 
Los valores mínimos de producción de fruto pudieron observarse en la dosis 0 de $\mathrm{N}$ (testigo) en los dos períodos de siembra (con 9,79 $\mathrm{Mg} \mathrm{ha}^{-1}$ en otoño/invierno y 6,00 $\mathrm{Mg} \mathrm{ha}^{-1}$ en primavera/verano). Se comparó el testigo con la dosis de $250 \mathrm{~kg} \mathrm{ha}^{-1}$, hubo una diferencia de 7,04 y 7,5 $\mathrm{Mg} \mathrm{ha}^{-1}$ para otoño/invierno y primavera/verano, respectivamente, lo que indica que la fertilización resultó en producciones significativas con las aplicaciones de $\mathrm{N}$.

A pesar de que la okra es considerada como una planta rústica, se percibe la ocurrencia de un efecto creciente y satisfactorio con incrementos hasta la dosis de $250 \mathrm{~kg} \mathrm{ha}^{-1} \mathrm{de} \mathrm{N}$, y se comportó como una planta de alta respuesta a este nutrimento, que afecta positivamente en la fructificación de la planta y contribuye para el desarrollo del sistema radical, producción y calidad de los frutos.

El tratamiento sin fertilización con $\mathrm{N}$ presentó productividad inferior a aquellos, con fertilización.

La masa del fruto varió de 16 a $20 \mathrm{~g}$, siendo la dosis de $250 \mathrm{~kg} \mathrm{ha}^{-1}$ la que presentó los valores más altos (20 g) en los dos períodos de siembra (Figura 3).

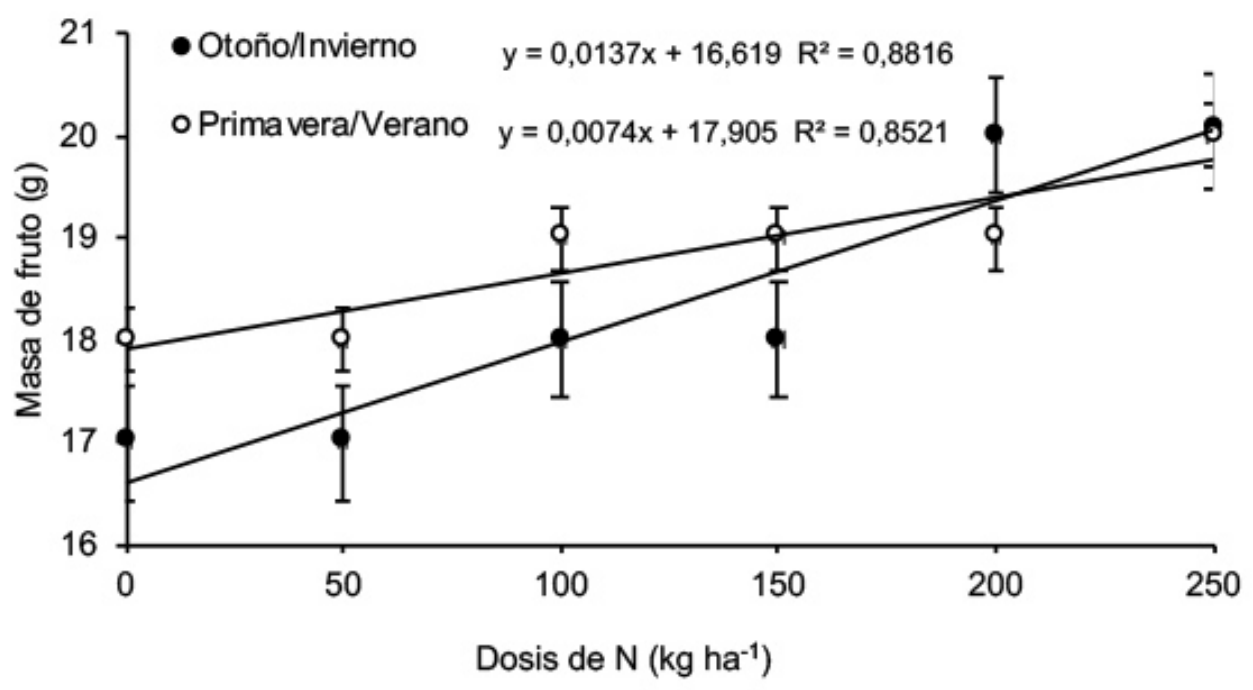

Figura 3. Masa del fruto de la okra (Abelmoschus esculentus L.), en función de la dosis de N. Campus Gurupi, Universidad Federal de Tocantins, Brasil. 2015.

Figure 3. Okra (Abelmoschus esculentus L.) fruit mass, depending on the $\mathrm{N}$ dose. Campus Gurupi, Universidad Federal de Tocantins. Brasil. 2015.

El testigo presentó masa media del fruto más baja con $16 \mathrm{~g}$ y $18 \mathrm{~g}$ en el período de otoño/invierno y primavera/ verano, respectivamente.

Para la altura de la planta se observó diferencia significativa entre los períodos de siembra y entre los tratamientos de N. En el cultivo de otoño/invierno la media fue de $92,46 \mathrm{~cm}$ y para primavera/verano fue de 71,06 cm.

La altura de las plantas se incrementó con el aumento de las dosis de $\mathrm{N}$, sin embargo, ese aumento no se reflejó en la masa media del fruto en el período de otoño/invierno, conforme se puede ser observar en la Figura 3. Se observó que el crecimiento de las plantas se relacionó con la productividad y en este sentido fue dependiente de la disponibilidad del N. Con $250 \mathrm{~kg} \mathrm{ha}^{-1}$ se obtuvo las mayores alturas de la planta con 97,95 (otoño/invierno) y 84,65 $\mathrm{cm}$ (primavera/verano) (Figura 4).

El índice de clorofila entre períodos de siembra no difirió entre sí. Sin embargo, el índice aumentó en relación a las dosis de N (Figura 5). 


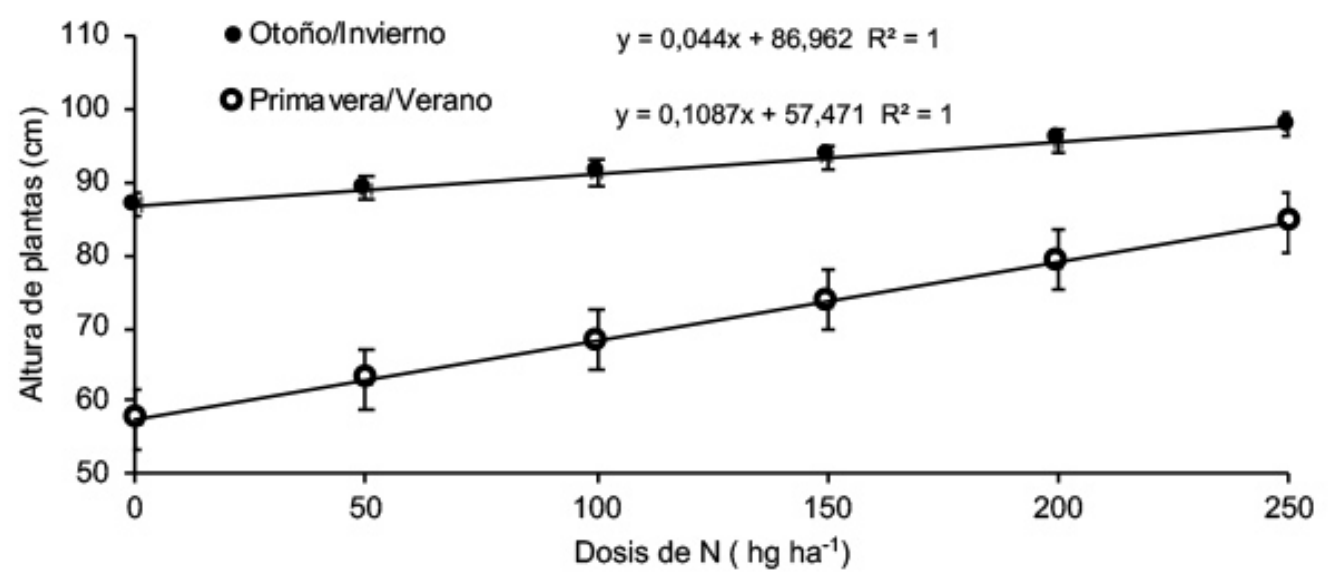

Figura 4. Altura de plantas de okra (Abelmoschus esculentus L.) en función de la dosis de N. Campus Gurupi, Universidad Federal de Tocantins, Brasil. 2015

Figure 4. Okra (Abelmoschus esculentus L.) plant height depending on the $\mathrm{N}$ dose. Campus Gurupi, Universidad Federal de Tocantins, Brasil. 2015.

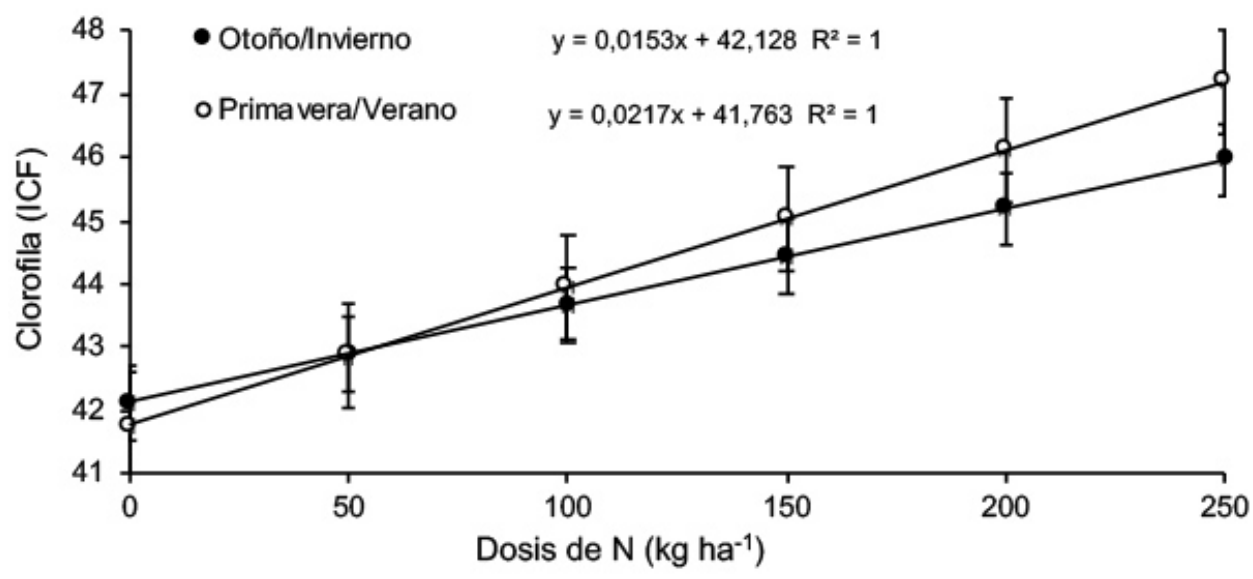

Figura 5. Índice de clorofila total en cultivo de la okra (Abelmoschus esculentus L.) en función de dosis de N. Campus Gurupi, Universidad Federal de Tocantins, Brasil. 2015.

Figure 5. Total chlorophyll index in okra (Abelmoschus esculentus L.) cultivation as a function of N dose. Campus Gurupi, Universidad Federal de Tocantins, Brasil. 2015.

Los índices de clorofila más altos fueron de 45,96 y 47,19 con $250 \mathrm{~kg} \mathrm{~N}^{-1}$ para el período otoño/invierno y primavera/verano, respectivamente. Los índices más bajos fueron para el testigo con 42,13 en el primer período y 41,76 en el segundo.

El índice de clorofila aumentó linealmente con el incremento de las dosis de $\mathrm{N}$. En la dosis de $250 \mathrm{~kg} \mathrm{ha}^{-1} \mathrm{el}$ índice de clorofila fue más alto. 


\section{Discusión}

La mayor producción de fruto se dio en otoño/invierno (13,86 $\left.\mathrm{Mg} \mathrm{ha}^{-1}\right)$ (Cuadro 1), estos resultados pueden atribuirse a las condiciones climáticas de cada período.

Los datos meteorológicos en la región durante los períodos de realización de los experimentos (Figura 1) registraron una temperatura máxima en otoño/invierno entre 31,6 a $34{ }^{\circ} \mathrm{C}$ y una humedad relativa que varió de 58,0 a $85,5 \%$, en tanto en el período de primavera/verano la temperatura máxima varió entre 34,8 a $38,7{ }^{\circ} \mathrm{C}$ con humedad relativa entre 51,8 a $77,2 \%$, lo que posiblemente promovió un efecto negativo en el desarrollo y producción en el cultivo de la okra.

Los factores meteorológicos pueden influenciar en el desarrollo y la producción, debido a que actúan directamente en el metabolismo de la planta (Taiz y Zeiger, 2013). La temperatura media más apropiada para el cultivo de la okra está entre 21,1 a $29,4^{\circ} \mathrm{C}$, con una media máxima en $35^{\circ} \mathrm{C}$ y una mínima de $18,3{ }^{\circ} \mathrm{C}$ (Sonnenberg y da-Silva, 2002). La humedad relativa para la producción de la okra también es importante, ya que la mayoría de las enfermedades fúngicas ocurre debido a la alta humedad, para la cultura de la okra, la cantidad de agua en el suelo no debe ser inferior a $65 \%$ de capacidad de campo (Embrapa, 1982). En los meses lluvioso, las pérdidas de $\mathrm{N}$ aplicado pueden acentuarse en prejuicio del desempeño agronómico de las hortalizas. Al mismo tiempo, la volatilización del $\mathrm{NH}_{3}$ de la urea aumenta con la temperatura, por lo que la taza de hidrólisis proporciona mayor cantidad de $\mathrm{NH}_{3}$ volátil (Longo y Melo, 2005; Duarte, 2006).

La producción de okra obtenida se encontró dentro del rango de la media brasileña que se estima en torno a 15 y $20 \mathrm{Mg} \mathrm{ha}^{-1}$, con un ciclo vegetativo que varía entre 150 y 400 días, y $12 \mathrm{Mg} \mathrm{ha}^{-1}$ cuando el ciclo es de 120 días (Donadelli et al., 2010; Galati et al., 2013). Resultados similares fueron encontrados por Oliveira et al. (2003), al estudiar la producción de la okra en la ciudad de Areia en el estado de Paraíba en función de la dosis de N (0, 50, 100, 150 y $200 \mathrm{~kg} \mathrm{ha}^{-1}$ ), donde observaron una producción máxima de $16,7 \mathrm{Mg} \mathrm{ha}^{-1}$ en la dosis de $\mathrm{N}$ de $150 \mathrm{~kg} \mathrm{ha}^{-1}$, y en la dosis 0 (testigo) una productividad máxima de $6 \mathrm{Mg} \mathrm{ha}^{-1}$.

El incremento en la producción ocurre debido a que la okra exige mayores cantidades de $\mathrm{N}$, siendo este nutriente limitante en su producción, principalmente en suelos tropicales, donde su contenido es bajo, lo que implica la práctica de fertilización nitrogenada. En el trabajo de Oliveira et al. (2013) también se relataron efectos positivos del $\mathrm{N}$ en la masa del fruto (18 g), número de frutos por planta (34 frutos planta-1) y en la producción (22 $\mathrm{Mg} \mathrm{ha}^{-1}$ ) de la okra. La masa del fruto máximo obtenido en este trabajo fue similar al de Zucchi et al. (2012) de 20 g. Al omitir el $\mathrm{N}$ en la solución nutritiva en la okra, se observó una reducción en la altura de las plantas y caída intensa de los botones florales, lo que redujo su fructificación (Costa et al., 1981).

La dosis máxima de $\mathrm{N}$ comparada con la ausencia de la fertilización nitrogenada resultó en la mayor masa del fruto, derivando en una mayor eficiencia de uso de los factores del medio que se tradujo en frutos con masa mayor (Duarte y Peil, 2010).

Una baja o falta del $\mathrm{N}$ en la planta provocaría una disminución de los asimilados, consecuentemente se disminuiría el número de frutos por planta, una reducción en la masa y otras mudanzas de las características del fruto, como es el largo, ancho, color y textura (Cardoso y Berni, 2011).

La deficiencia de $\mathrm{N}$ en el cultivo afecta y limita el crecimiento y desarrollo vegetativo, reduce el tamaño de las hojas por la falta de clorofila, baja formación de proteínas y materiales genéticos estructurales, lo que disminuye el proceso de fotosíntesis (Heckman, 2002).

La altura máxima de la planta fue de $98 \mathrm{~cm}$ en la mayor dosis de $\mathrm{N}$, otros autores como Nascimento et al. (2015) reportaron valores inferiores al utilizar diferentes niveles de salinidad en la irrigación y cuatro dosis de $\mathrm{N}$ $\left(50,75,100\right.$ y $\left.125 \mathrm{~kg} \mathrm{ha}^{-1}\right)$ en Cruz de las Almas - BA con una altura máxima de 75,16 cm; en ese mismo sentido Costa (2014), en Santa Helena de Goiás - GO, observó una altura máxima de 73,82 cm.

En relación con el índice de clorofila, Sediyama et al. (2009) obtuvieron resultados similares a los de la 
presente investigación, al evaluar la productividad de la okra en función de dosis de biofertilizante porcino en Oratórios - MG, donde encontraron un incremento lineal al aumentar la dosis de biofertilizante, observaron con la dosis máxima un índice de clorofila de 46,87. El mayor índice ocurrió debido a una mayor cantidad de pigmento presente en la hoja, ya que este se correlaciona positivamente con el contenido de $\mathrm{N}$ en las plantas, principalmente al hecho de que entre 50 y $70 \%$ del $\mathrm{N}$ total en las hojas son parte integrante de enzimas que estan asociadas a los cloroplastos. El índice de clorofila puede ser indicativo de la concentración de $\mathrm{N}$ en las hojas, ayudando en el manejo de la fertilización nitrogenada de los cultivos (Embrapa, 2012).

Así como la producción aumentó linealmente con el incremento de las dosis de $\mathrm{N}$, el índice de clorofila respondió de la misma manera, ya que la concentración de $\mathrm{N}$ tiene relación directa con la eficiencia fotosintética. Esto ocurre por el hecho de la clorofila es responsable por la conversión de energía luminosa a energía química por fotoreceptores específicos (Larcher, 2000). El proceso ocurre en las hojas por la dependencia de enzimas, constituidas básicamente de proteínas, que dependen del N (Smeal y Zhang, 1994; Larcher, 2000).

\section{Conclusiones}

La época de siembra y el contenido de nitrógeno en el suelo interactuaron con la altura de la planta de okra, siendo favorable en el periodo que no ocurrió precipitación.

El $\mathrm{N}$ puede influenciar en todas las características, lo que demuestra la importancia de la fertilización nitrogenada en el desarrollo de las plantas en el cultivo de la okra.

\section{Literatura citada}

Adejoye, O., J. Awokoya, and E. Oluseyi. 2009. Effect of seasonal changes on growth and yield of okra (Abelmoschus esculentus (L.) Moench). Res. J. Agric. Biol. Sci. 5:940-943.

Alvarado, C.M., F.A. Díaz, y C.I. Garza. 2007. Micorrización de okra (Abelmoschus esculentus L.) en riego por goteo. Rev. Fitotec. Mex. 30:437-441.

Argenta, G., P.R.F. da-Silva, e C.G. Bortolini. 2001. Clorofila na folha como indicador do nível de nitrogênio em cereais. Ciênc. Rural 31:715-722. doi:10.1590/S0103-84782001000400027

Bredemeier, C., e C.M. Mundstock. 2000. Regulação da absorção e assimilação do nitrogênio nas plantas. Ciênc. Rural 30:365372. doi:10.1590/S0103-84782000000200029

Cardoso, M.O. 2001. Desempenho de cultivares de quiabo em condições de terra firme do estado do Amazonas. Apresentado em: Congresso Brasileiro de Olericultura, 41. 1er Encontro sobre plantas medicinais, aromáticas e condimentares, Brasília, BRA. 2 jul. 2001. Paper 018.

Cardoso, M.O., e R.F. Berni. 2011. Crescimento e produção em quiabeiro com doses de nitrogênio. Hort. Bras. 29:1777-1783.

Cerri, A.M., y F. Vilella. 1996. Generación del rendimiento de okra: Comparación de dos cultivares e dos fechas de siembra. Rev. Fac. Agron. 15(2-3):207-212.

Chapman, S.C., and H.J. Barreto. 1997. Using a chlorophyll meter to estimate specific leaf nitrogen of tropical maize during vegetative growth. Agron. J. 89:557-562. doi:10.2134/agronj1997.00021962008900040004x

Costa, M.C.B., G.D. Oliveira, e H.P. Haag. 1981. Nutrição mineral de hortaliças. Efeito da omissão dos macronutrientes e do boro no desenvolvimento e na composição química do quiabeiro. Em: H.P. Haag, e K. Minami, editores, Nutrição mineral em hortaliças. Fundação Cargill, Campinas, BRA. p. 257-275. 
Costa, R.A. 2014. Cultura do quiabo submetida a lâminas de irrigação por gotejamento em função da evaporação em tanque classe A. Dr. Diss., Universidad Estatal Paulista, São Paulo, BRA.

Dilruba, S., M. Hazanuzzaman, R. Karim, and K. Nahar. 2009. Yield response of okra to different sowing time and application of growth hormones. J. Hortic. Sci. Ornam. Plants 1(1):10-14.

Donadelli, A.P., H.N. Turco, C. Kano, S.W. Tivelli, e L.F.V. Purquerio. 2010. Rentabilidade e custo de produção do quiabeiro consorciado com adubos verdes. Hort. Bras. 28:411-415.

Duarte, F.M. 2006. Perdas de nitrogênio por volatilização de amônia e eficiência de adubação nitrogenada na cultura do arroz irrigado. MSc. Diss., Universidade Federal de Santa Maria, Rio Grande do Sul, BRA.

Duarte, T.S., e R.M.N. Peil. 2010. Relações fonte: dreno e crescimento vegetativo do meloeiro. Hort. Bras. 28:271-276.

Embrapa (Empresa Brasileira de Pesquisa Agropecuária). 1982. Sistema de produção para a cultura do quiabo. Série Sistema de Produção $n^{\circ}$ 004. Embrapa, BRA.

Embrapa (Empresa Brasileira de Pesquisa Agropecuária). 2012. Rendimento do quiabeiro com doses de nitrogênio em cultivo não adensado. Boletim de Pesquisa e Desenvolvimento 15. Embrapa, Amazônia Ocidental, BRA.

Embrapa (Empresa Brasileira de Pesquisa Agropecuaria). 2013. Sistema brasileiro de classificação de solos. 3er ed. Embrapa, Brasília, BRA.

Ferreira, D.F. 2008. SISVAR-Sistema de análise de variância. Versão 5.3. Universidade Federal de Lavras, Minas Gerais, BRA.

Filgueira, F.A.R. 2000. Novo manual de olericultura: Agrotecnologia moderna na produção e comercialização de hortaliças. Universidade Federal de Viçosa, Viçosa, BRA.

Galati, V.C., V.C. Galati-Filho, e A.U. Alves. 2013. Crescimento e acúmulo de nutrientes da cultura do quiabeiro. Semin. Ciênc. Agrár. 34:191-199. doi:10.5433/1679-0359.2013v34n1p191

Gonçalves, G.C. 2008. Cultura do quiabo. Jornal Agrícola, BRA. http://jornalagricola.wordpress.com/2008/02/24/cultura-doquiabo/ (acesso 22 fev. 2016).

Heckman, J.R. 2002. In-season soil nitrate testing as a guide to ntrogen management for annual crops. Hort. Technol. New Brunswick 12:706-710. doi:10.21273/HORTTECH.12.4.706

INMET (Instituto Nacional de Meteorología). 2015. Mapas do Boletim Agro-climatológico. INMET, BRA. http://www.inmet. gov.br/portal/index.php?r=agrometeorologia/boletimAgroclimatologico (acesso 22 fev. 2016).

Köppen, W.P. 1948. Climatología: con un estudio de los climas de la tierra. Fondo de Cultura Económica, México D.F., MEX.

Larcher, W. 2000. Ecofisiologia vegetal. RiMa Editora, São Carlos, BRA.

Longo, R.M., e W.J. Melo. 2005. Hidrólise da ureia em latossolos: efeito da concentração de ureia, temperatura, pH, armazenamento e tempo de incubação. Rev. Bras. Ciênc. Solo 29:651-657. doi:10.1590/S0100-06832005000400018

Malavolta, E. 2008. O futuro da nutrição de plantas, tendo em vista aspectos agronômicos, econômicos e ambientais. Inform. Agron. Piracicaba 121:1-10.

Marcussi, F.F.N., L.J.G. Godoy, e R.L. Villas-Bôas. 2004. Fertirrigação nitrogenada e potássica na cultura do pimentão baseada no acúmulo de N e P pela planta. Irriga 9:41-51. doi:10.15809/irriga.2004v9n1p41-51

Nascimento, P.S., V.P.S. Paz, L.S. Fraga-Junior, e I.P. Costa. 2015. Crescimento vegetativo do quiabeiro em função da salinidade da água de irrigação e da adubação nitrogenada. Colloq. Agrar. 13:10-15. doi:10.5747/ca.2017.v13.n1 .a143

Oliveira, A.P., A.U. Alves, C.S.M. Dornelas, J.A. Silva, e M.L. Porto. 2003. Rendimento de quiabo em função de doses de nitrogênio. Acta Sci. Agron. 25:265-268. doi:10.4025/actasciagron.v25i2.1761 
Oliveira, A.P., N.A. Oliveira, O.P.R. Silva, S.M. Pinheiro, e A.D.G. Neto. 2013. Rendimento do quiabo adubado com esterco bovino e biofertilizante. Sem. Ciênc. Agrár. 3:2629-2636. doi:10.5433/1679-0359.2013v34n6p2629

Ossomon, E.N., and V.N. Kunene. 2011. Effects of planning dates on seedling emergence and vigor of Okra (Abelmoschus esculentus (L) Moench) in Swaziland World. J. Agric. Sci. 7:320-326.

Sediyama, M.A.N., M.R. Santos, S.M. Vidigal, L.T. Salgado, M.W. Pedrosa, e L.L. Jacob. 2009. Produtividade e estado nutricional do quiabeiro em função da densidade populacional e do biofertilizante suíno. Bragantia 68:913-920. doi:10.1590/S0006-87052009000400011

Smeal, D., and H. Zhang. 1994. Chlorophyll meter evaluation for nitrogen management in corn. Communications in Soil Science and Plant Analysis, New York, 25 (9/10):1495-1503.

Sonnenberg, P.E., e N.F. da-Silva. 2002. Desenvolvimento e produção do quiabeiro em função das datas de plantio. Pesqui. Agropecu. Trop. 32:33-37. doi:10.5216/pat.v32i1.2438

Supatra-Sen, S.M., and S. Sen. 1998. Influence of seasons in determining the date of sowing and fruit quality of Abelmoschus esculentus (L.) Moench (okra) and Lycopersicon esculentum Mill. (tomato). Indian Agric. 43(3):161-166.

Taiz, L., y E. Zeiger. 2013. Fisiología vegetal. 5ta ed. Artmed, MA, USA.

Tukey, J.W. 1949. Comparing individual means in the analysis of variance. Biometrics 5:99-114. doi:10.2307/3001913

Zucchi, M.R., F.K. Perinnazzo, N. Peixoto, W.R. Mendanha, e M.A. Zatarin. 2012. Associação das culturas de quiabo e feijãocaupi. Rev. Agrotecnol. 3(2):12- 23. 